\title{
Evaluation of Trauma Management in Injured Iranian Hajj Pilgrims in 2015 Mina Stampede
}

\author{
Shaban Mehrvarz ${ }^{1}$, Mohammad Javad Bagheri ${ }^{1}$, Shahram Manoochehry ${ }^{1,}{ }^{*}$, Behzad Einollahi ${ }^{2}$, Mohammad Ganjeh $^{1,3}$, \\ Taher Doroudi ${ }^{3,4}$ and Seyyed Ali Marashi ${ }^{3}$
}

1 Trauma Research Center, Baqiyatallah University of Medical Sciences, Tehran, Iran

2 Nephrology and Urology Research Center, Baqiyatallah University of Medical Sciences, Tehran, Iran

${ }^{3}$ Hajj Medical Center, Iranian Red Crescent Society, Tehran, Iran

4 Shefa Neuroscience Research Center, Khatam-al-Anbia Hospital, Tehran, Iran

* Corresponding author: Shahram Manoochehry, Trauma Research Center, Baqiyatallah University of Medical Sciences, Tehran, Iran. Tel: +982181263582; Email: shahram.manoochehry@yahoo.com

Received 2020 December 16; Revised 2021 February 01; Accepted 2021 February 15.

\begin{abstract}
Background: The Muslim annual pilgrimage (Hajj) ceremony in 2015 was one of the most catastrophic and tragic events of the Hajj pilgrimage in history. Thousands of pilgrims have been tragically stampeded to death and injured in the land of Mina in Mecca, Saudi Arabia. The last official statistics declared that at least 2,431 pilgrims died and 427 pilgrims were missed in the blocked street. Furthermore, 465 corpses were Iranian pilgrims.

Objectives: The purpose of this study was to evaluate the treatment of the injured pilgrims by the Iranian Hajj Medical Team in Mina and assess its strengths and weaknesses in order to develop a comprehensive approach in dealing with similar events.

Methods: The data were extracted from the records of the patients obtained from the archives of the Islamic Republic of Iran Hajj Medical Center. Some interviews were performed with the members of the medical team who were present at the incident scene. Finally, a protocol was proposed for the management of patients in similar incidents.

Results: Out of a total of 1,633 referred patients, 567 injured pilgrims were admitted and treated during the incident. A total of 403 complete medical sheets were included in this study. Moreover, 362 cases $(89.8 \%)$ improved after cooling and proper fluid replacement. They were discharged with therapeutic measures. A total of 41 injured pilgrims $(10.2 \%)$ were referred to urban hospitals, and 13 cases of the referrals were due to acute respiratory distress syndrome. Furthermore, 12 subjects were referred for dialysis, and 4 cases of the referrals were due to anxiety disorders. Moreover, three cases of diabetic ketoacidosis, one case of epidural hemorrhage, one case of intracranial hemorrhage, one case of spleen hematoma, one case of sigmoid volvulus, and one case of gastrointestinal bleeding were successfully treated. The most common diagnosis was dehydration, followed by heat exhaustion and contusion. Approximately, all the victims had some degrees of the aforementioned injuries. Initial therapeutic attempts included rehydration, cooling in the cold tunnel, and correction of electrolyte imbalances. Performing a complete blood count with differential, blood urea nitrogen, sodium, and potassium tests.-Urinary catheterization was determined as per case; nevertheless, fluid therapy and cooling were carried out for all the injured patients.

Conclusion: Therapeutic efficacy was evaluated as excellent due to zero mortality. As a result, it was decided to propose a treatment method for the Hajj Medical Team as a protocol for the management and treatment of injuries caused by mass gathering (e.g., contusion and heat exhaustion) in similar events.
\end{abstract}

Keywords: Hajj, Heat exhaustion, Mina accident, Overcrowding, Stampede

\section{Background}

More than 2 million Muslim pilgrimages gather annually for the Hajj ceremony. The 2015 Hajj ceremony was one of the most deadly and tragic events of the Hajj pilgrimage in history. On September 24 in 2015, at least 2,431 pilgrims died and 427 were reported missing after overcrowding of huge mass of individuals in the blocked street of Mina in Mecca, Saudi Arabia, leading the pilgrims to the Jamaraat Bridge. In addition, 465 corpses were Iranian pilgrims (1). To date, there have been no official statistics on the injured patients on the same day in other medical centers in Mina; however, 1,633 patients were evacuated to the Iranian Hajj Medical Center. On the day of the accident, the temperature was higher than $50^{\circ} \mathrm{C}$.

Mass gathering and overcrowding occur at certain religious and social ceremonies, such as Hajj, Arba'een, Ashura ceremonies, or some political, cultural, and sports gatherings, in which a large community of individuals gathers in a limited time and place. Due to the multiplicity and repetition of these ceremonies every year, special concerns are raised. In these situations, a sudden excitation due to an unforeseen incident can rapidly restrain the transfer of a large number of individuals to a restricted location and events, such as the 2015 Mina stampede, as mentioned in previous studies (1-3). Considering the history and probability of the occurrence of such events, different studies have been carried out on various dimensions in order to prevent adverse events $(1,2)$.

In recent years, Hajj ceremonies have coincided with a very high temperature and large crowding of pilgrims in a short period of time in a limited area 
(Mina). In the event of overcrowding during the ceremony, in addition to stampede, severe heat exhaustion can lead to increased morbidity and mortality (4-7). An important point is the repeatability of these tragic events, which further elaborates on the need to address this issue (8) since any misunderstanding in the management, support, and healing is likely to cause disasters and injuries or mortalities for many individuals (9-11). Therefore, further investigations require an in-depth understanding of the causes of injuries and casualties in these cases, especially Hajj ceremonies, and techniques for the prevention of accidents and mortality and morbidity caused by overcrowding (12).

\section{Objectives}

This study was designed to examine the types of traumatic injuries and evaluate the treatment process of victims in the tragic stampede event of 2015 at different levels. In other words, the current study sought to provide a solution for reducing the number of casualties and injuries in similar events. Accordingly, the present study aimed to evaluate the treatment of the injured pilgrims in the Iranian Hajj Medical Center and assess its strengths and weaknesses in order to offer a comprehensive approach in dealing with similar events.

\section{Methods}

The present study investigated the medical records of 567 admitted patients in the archives of the Islamic Republic of Iran Hajj Medical Center. Firstly, the data, including demographic information, method of triage, cause of admission, treatment methods, and referral to Mecca hospitals (i.e., Iranian or Saudi), were extracted and classified. Afterward, interviews with some of the medical team members were carried out to complete some incomplete or obscure data. Furthermore, a questionnaire was designed to assess their ideas about the quality of medical care in that situation and propose and develop a protocol using the Delphi method. Finally, the information on the treatment records of 403 patients was complete and useful and included in the current study.

The quality of medical team operations was evaluated and the results are shown in Table 3 . The names of Hajj medical staff were collected from the Medical Center of Iranian Red Crescent Society. Finally, 19 members of the staff (including 8 physicians, 7 nurses, and 4 health care staff members) participated in this study.

For the development of an appropriate protocol, a questionnaire was prepared based on the viewpoints of key practitioners and experts of the medical team and presented to experts in the field, where they were asked to answer questions. After receiving the filled forms, the answers were reviewed and categorized, followed by the prioritization of the items. Then, the sheets containing a complete list of events, functions, and suggestions were sent to all the previous experts to score the importance of each item. Finally, the treatment protocol was proposed according to experts' consensus. Frequency and mean percentage, standard deviation, median, quadratic amplitude, and amplitude were used to describe the data. A 95\% confidence interval was utilized to express the accuracy of the estimates. All the analyses were carried out using SPSS statistical software (version 20.0), and a p-value of less than 0.05 was considered statistically significant.

\begin{tabular}{|c|c|c|c|}
\hline Item & Example(s) & $\begin{array}{c}\text { Score } \\
(1-10)\end{array}$ & Comment \\
\hline Primary evacuation & Speed and safety of transfer & 9.1 & Excellent \\
\hline Triage & Vital signs and priority for transfer of alive cases (not transferring the dead pilgrims) & 8.7 & Very good \\
\hline Quality of medical care & Intravenous solutions, ventilation support, and cooling & 9 & Very good \\
\hline Adequacy of facilities & Ambulances, beds, and monitoring & 4.1 & Weak \\
\hline Management of scene & Motivation, experience, and coordination & 10 & Excellent \\
\hline
\end{tabular}

\section{Results}

A total of 1,633 patients were evacuated to the Iranian Hajj Medical Center in Mina, out of whom 567 cases were admitted (403 cases of them with complete available data enrolled in the study) and 1,066 cases were treated as outpatients. The mean age of the admitted patients was 53.7 years, within the range of 24-73 years (Table 1 ).

According to the results of the present study and interviews with the medical staff involved in the scene, the incident was unofficially reported to them.
In addition, from the very early minutes of the stampede, the rescue groups of the Iranian medical team (including eight physicians, seven experienced nurses, and four health care staff members) were dispatched to the scene. In the first hours before the arrival of the Saudi military forces, rescue operations, including evacuation and resuscitation of the injured patients, were performed by the Iranian rescue team. The evacuation process took place by foot or ambulance within less than $1 \mathrm{~km}$ distance from the scene of the accident to the Emergency Department.

There were two cold tunnels in the Emergency 


\begin{tabular}{lccc}
\hline \multicolumn{2}{l}{ Table 1. Evaluation of information records of patients in Mina Emergency Clinic in Mecca, Saudi Arabia } & \\
\hline Mina Emergency Clinic & Diagnosis & Frequency & $\begin{array}{c}\text { Frequency } \\
\text { percentile }\end{array}$ \\
\hline & Anuria & 15 & $3.7 \%$ \\
& Contusion & 385 & $85 \%$ \\
& 403 & $100 \%$ \\
Mina Emergency managements & Dehydration & 403 & $100 \%$ \\
Criteria for referrals to urban hospitals & heat stroke or Heat exhaustion & 403 & $100 \%$ \\
Discharge criteria & Rehydration & 403 & $100 \%$ \\
Mortality in Mina emergency & Cooling & 41 & $10.2 \%$ \\
\hline & Conscious, normal laboratory tests, and adequate urinary output & 362 & $89.8 \%$ \\
\hline
\end{tabular}

Department of Mina hospital each of which was constructed by the interconnection of multiple tents, air conditioners, and sprinkler fans. Furthermore, icemakers were installed in tunnels and a large amount of cold intravenous (IV) fluids were available there. It is worth mentioning that although there was a huge stream of injured individuals during the incident time, due to the proper primary triage and rapid turnover of patients in the Emergency Room, no patient was deprived of medical care and the injured cases were immediately taken to the hospital after the initial resuscitation or dismissed and delivered to the physicians of the Hajj groups.

Out of 403 studied cases, 362 subjects (89.8\%) were improved and discharged with therapeutic measures. The most common early diagnoses were dehydration caused by heat stroke (or heat exhaustion) and contusion which all the patients had to some extent. The initial measures included IV cold fluid therapy, cooling, and correction of electrolyte imbalance that were performed for all patients. A complete blood count with differential, blood urea nitrogen, sodium, and potassium tests, and urinary catheterization were carried out on a case-by-case basis. It is worth mentioning that in rather stable patients with mild dehydration, heat stroke, and rather stable vital signs, resuscitation was performed by means of a cold tunnel, ice bath, and oral or IV administration of cold fluids on an outpatient basis.

The patients with more critical conditions, such as a loss of consciousness, hypotension, anuria, traumatic injuries, and severe heat stroke, were admitted to the hospitals. Urinary catheterization was carried out for them and they were treated with cold IV normal saline or half-normal saline and, if necessary, IV mannitol. Moreover, when their conditions became stable, they were discharged and delivered to the Hajj group and the treatment would continue under the supervision of the physicians in the group. The presence of group physicians was a major contributor to the treatment of the injured individuals and reduced the workload of the Medical Team at the Emergency Department of Mina hospital. Out of a total of 403 existing cases admitted to the Emergency Department of Mina Field Clinic, 362 cases had a rather stable general condition and were discharged after proper resuscitation with normal laboratory data and adequate urine output. Furthermore, 29 patients were transferred to the Iranian hospital in Mecca and 12 cases were referred to the Saudi Hospital in Mecca (altogether a total of 41 cases [10.2\%]).

According to Table 2, 13 cases $(3.2 \%)$ of the referrals due to acute respiratory distress syndrome underwent intubation and were transferred to the Saudi Hospital in Mecca. In addition, 12 cases due to high serum creatinine levels and rhabdomyolysis were referred to an urban hospital in Mecca for dialysis. Other referral cases included anxiety disorders for psychiatric counseling $(\mathrm{n}=4)$, diabetic ketoacidosis $(n=3)$, epidural hemorrhage $(n=1)$, intracranial hemorrhage undergoing neurosurgery operation $(n=1)$, spleen hematoma leading to splenectomy $(n=1)$, and sigmoid volvulus which was

\begin{tabular}{|c|c|c|c|}
\hline Urban hospital (Mecca) & Ideas & Frequency & Frequency percentile \\
\hline \multirow{9}{*}{$\begin{array}{l}\text { Reason for admission } \\
\text { and referral }\end{array}$} & Rhabdomyolysis (Dialysis) & 12 & $3.0 \%$ \\
\hline & Acute respiratory distress syndrome (Intubation) & 13 & $3.2 \%$ \\
\hline & Anxiety disorder & 4 & $1.0 \%$ \\
\hline & Diabetic ketoacidosis & 3 & $0.7 \%$ \\
\hline & Epidural hemorrhage & 1 & $0.2 \%$ \\
\hline & Intracranial hemorrhage & 1 & $0.2 \%$ \\
\hline & Splenic hematoma & 1 & $0.2 \%$ \\
\hline & Sigmoid volvulus & 1 & $0.2 \%$ \\
\hline & Gastrointestinal bleeding & 1 & $0.2 \%$ \\
\hline \multirow{5}{*}{ Therapeutic measures } & Dialysis & 12 & $3.0 \%$ \\
\hline & Gastrointestinal surgery & 1 & $0.2 \%$ \\
\hline & Splenectomy & 1 & $0.2 \%$ \\
\hline & Neurosurgery & 2 & $0.5 \%$ \\
\hline & Consultation with psychologist & 4 & $1.0 \%$ \\
\hline Mortality & Death in the patients referring to emergency department & 0 & $0 \%<$ \\
\hline
\end{tabular}


operated in the Saudi Hospital (n=1), and gastrointestinal bleeding that was conservatively treated $(n=1)$.

\section{Discussion}

The issues, including overcrowding, mass gathering, and adverse outcomes of overcrowdingdriven events, in holy places have affected the process of rescue and treatment, leading to reducing the efficiency of medical staff. This requires a more detailed study in order to optimize crisis management $(9,10)$. In the present study, in addition to the assessment of the rescue and treatment process of the patients in the Mina incident, the management system and strengths and weaknesses of all the components involved in the rescue and treatment process have been addressed to propose new strategies and protocols for the reduction of mortality and morbidity.

Yezli et al. proposed that the redefinition of physical and civil engineering infrastructure at the field is the most important preventive measure for the reduction of events, such as stampedes (11). It is obvious that Saudi Arabia should provide more appropriate infrastructure and architecture to prevent morbidity and morbidity. One of the important factors in the provision of health care services is the reliable and correct collection of information in the shortest time. This requires the crisis records of overcrowding in a simple, imaginative, functional, and illustrative manner; therefore, important information and red flags are prioritized. As proposed by Shinchi et al., the design of a suitable case scenario is essential in disruptive events (12).

Glazer et al. notifies the importance of rapid cooling in overcrowding events and proposed that rapid cooling has a significant role in the reduction of mortality and mortality $(13,14)$. In the present study, all the patients were affected by heat stroke (thermal exhaustion) and were dehydrated. Rapid cooling using existing equipment, such as air conditioners, creation of a cold tunnel, and cold infusion or oral fluids, saved most of the patients from renal failure and mortality. As noted, the time and speed of the initial triage at the scene of the accident are among the most important determinants of the reduction of mortalities caused by overcrowding (15), where a team of experienced physicians and nurses is necessary to provide the initial urgent triage at the scene of the final event.

Unfortunately, reliable data from the urgent triage at the scene of the Mina incident were not available; however, the eyewitnesses of the medical team reported that the Saudi forces blocked continuing the triage after their arrival in Mina, and this might have been one of the reasons of the high mortality at the tragedy scene. At the next level of triage in the hospital or clinic, the triage of transmitted patients was performed by a trained physician, followed by patient transmission to the cold tunnel or emergency ward and/or intensive care unit according to the severity and types of the injuries.

Outpatient measures, such as the use of ice bags and cold liquids, were used. After the stabilization of the general conditions of the patients, the physicians in the Hajj groups would accept them to continue the treatment. The physicians in the Hajj groups played a very important role in the treatment of these patients after discharge. The cases with more critical conditions were more intensively treated under the close supervision of the specialists and medical staff in the Iranian hospital of the Haj Medical Center in Mecca. These patients needed IV fluid therapy with normal or half-normal saline and urinary catheterization. A fluid intake and output chart was used for the evaluation of the adequacy of fluid therapy.

In patients with more critical conditions, IV mannitol was also infused. The use of ice bags, cooling blankets, and cold fluids should be taken into consideration and not neglected. In addition, gastric and bladder gavage with cold fluids was conducted for patients with higher degrees of heat stroke via nasogastric tubes and folly catheters. Laboratory tests, such as serum sodium, potassium, creatinine, lactate dehydrogenase, and creatinine phosphokinase, were carried out in the hospitalized patients to monitor and evaluate their conditions. Despite the first measures, if patients exhibited hyperthermia, anuria, and decreased unconscious level or deteriorated laboratory data, they were referred to an urban hospital for continued medical care.

Regarding the results, heat stroke, dehydration, and contusion were observed to be the most common causes of patient admission $(\mathrm{n}=403)$, indicating the importance of the weather conditions of the pilgrimage sites; as a result, cooling and hydration equipment should be considered beforehand (14). In addition, one of the most important offered services was hemodialysis. The need for further hemodialysis equipment and coordination and preparation of the necessary personnel in the emergency department is observed to perform dialysis for the cases in order to reduce the mortality and morbidity rates of such incidents. It should be taken into account that no mortality was observed among the admitted patients in the emergency department. This could show the importance of faster initiation of rescue and resuscitation measures that were prevented by the Saudi forces to save the injured individuals and reduce the mortality of the victims.

\section{Limitations}

The current study had several limitations. Firstly, the study was retrospective; therefore, most of the injured pilgrims were not available for interview and follow-up. Secondly, some Hajj Medical Organization physicians did not cooperate to interview and 
complete the questionnaire. Finally, the medical records of the injured patients in the Emergency Ward of Mina hospital were incomplete.

\section{Conclusion}

Therapeutic efficacy was evaluated as excellent due to zero mortality and very good management in spite of poor equipment. As a result of the present study, a heat exhaustion management protocol (Algorithm 1) was proposed for use in similar events (e.g., overcrowding and stampede). By announcing the alarm code, a pre-trained medical team starts initial triage and the rapid evacuation of alive

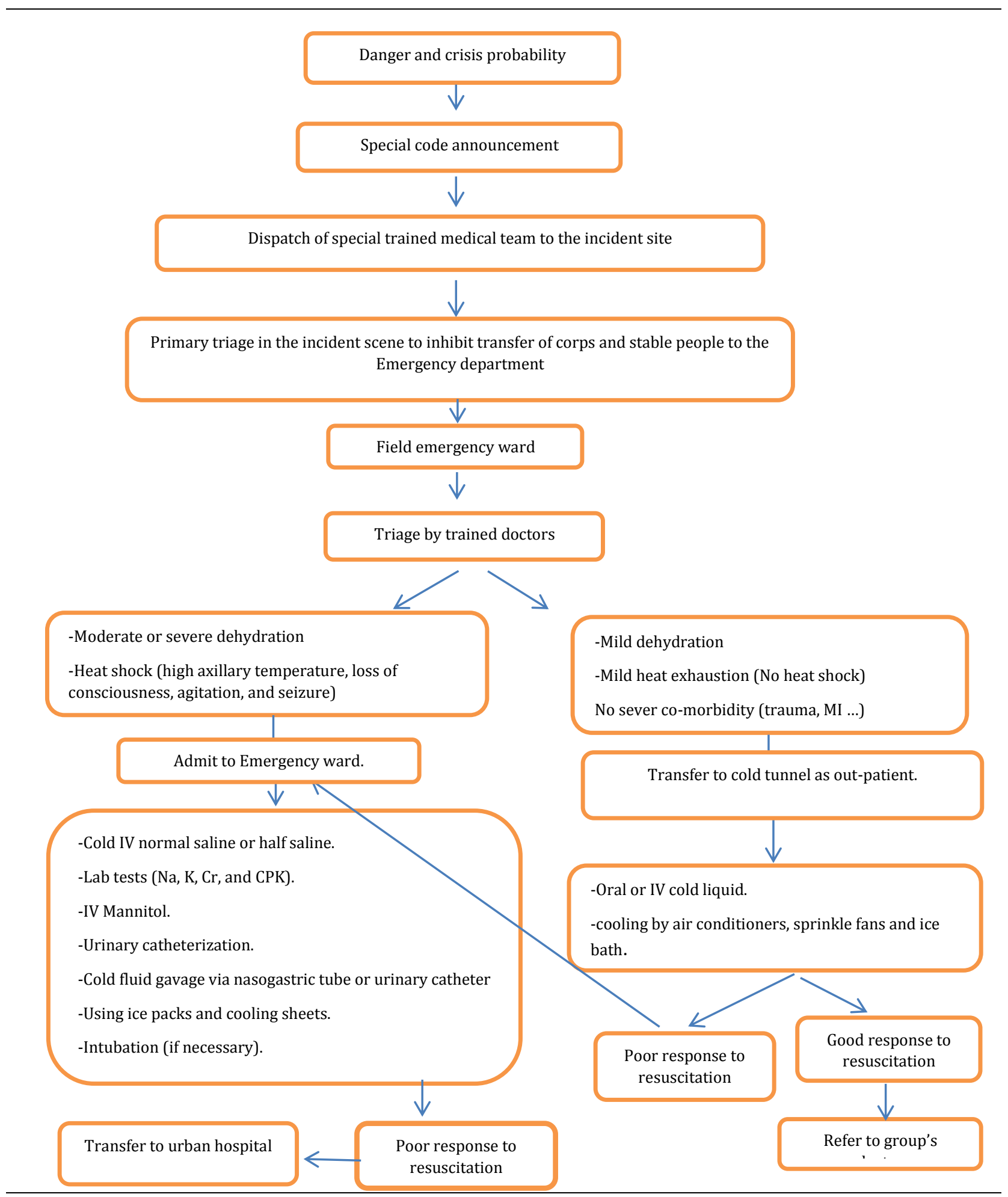

Algorithm 1. Suggested protocol for management of heat exhaustion in Emergency Ward of Mina Emergency Clinic 
pilgrims to health centers is of great importance; therefore, rescue operations, including proper medical care, at the emergent time would be of great importance in the field, leading to referring less injured individuals to urban hospitals.

\section{Acknowledgments}

We would like to thank the deputy research director of Baqiyatallah Hospital for their kind cooperation in this research.

\section{Footnotes}

Authors' Contribution: Study concept and design: ShabanMehrvarz

Acquisition of data: Mohammad javad Bagheri

Analysis and interpretation of data: Mohammad javad Bagheri, Shahram Manoochehry

Drafting of the manuscript: Mohammad javad Bagheri, Shahram Manoochehry

Critical revision of the manuscript for important intellectual content: Shaban Mehrvarz, Shahram Manoochehry

Behzad Einollahi, Mohammad Ganjeh

Statistical analysis: Mohammad javad Bagheri

Administrative, technical, and material support: Mohammad Ganjeh,Taher Doroudi ,Seyyed ali Marashi Study supervision: Shaban Mehrvarz

Conflict of Interests: We declare that there is not any conflict of interest in this study.

Ethical Approval: The protocol of the study was approved in the ethical committee of Baqiyatallah University of Medical sciences under IR.BUMS. REC.1395.127 cod.

Funding/Support: Not applicable.

Informed consent: Not applicable.

\section{References}

1. Ganjeh M, Einollahi B. Mass fatalities in Hajj in 2015. Trauma
Mon. 2016;21(5):e43253. doi: 10.5812/traumamon.43253. [PubMed: 28184365].

2. Manoochehry S, Rasouli HR. Recurrent human tragedy during Hajj. Int J Travel Med Glob Health. 2017;5(1):36-7. doi:10.15171/ijtmgh.2017.07.

3. Salamati P, Rahimi-Movaghar V. Hajj stampede in Mina, 2015: need for intervention. Arch Trauma Res. 2016;5(2):e36308. doi: 10.5812/atr.36308. [PubMed: 27703961].

4. Hoseinpourfard M, Ghanei M, Tofighi S, Ayoubian A, Izadi M. The emergence of Hajj stampedes: lessons for Hajj trauma centers. Trauma Monthly. 2017;22(4):E39455. doi: 10.5812/ traumamon.39455.

5. Khorasani-Zavareh D, Dadgari F, Karampourian A, Nouri F. Deaths and injuries in Hajj: An important implication for mass gathering management. Trauma Monthly. 2017;22(5):E40790. doi: 10.5812 /traumamon.40790.

6. Puriatarza E, Kamso S. Cause of death of indonesia Hajj in Armenia, Saudi Arabia 2015. KnE Life Scie. 2019;4(10):115-22. doi: $10.18502 / \mathrm{kls} . v 4 i 10.3776$.

7. Tufail A. Pilgrim tracking and location based services using RFID and Wireless sensor networks. Int J Computer Sci Network Secur. 2018;18(6):112-9.

8. Johansson A, Batty M, Hayashi K, Al Bar O, Marcozzi D, Memish ZA. Crowd and environmental management during mass gatherings. Lancet Infect Dis. 2012;12(2):150-6. doi: 10.1016/S1473-3099(11)70287-0. [PubMed: 22252150].

9. Shujaa A, Alhamid S. Health response to Hajj mass gathering from emergency perspective, narrative review. Turk J Emerg Med. 2015;15(4):172-6. doi: 10.1016/j.tjem.2015.02.001. [PubMed: 27239622].

10. Arnold JL, Paturas J, Rodoplu Ü. Measures of effectiveness of hospital incident command system performance. Prehosp Disaster Med. 2005;20(3):202-5. doi: 10.1017/s1049023x0 0002478. [PubMed: 16018512].

11. Yezli S, Khan A, Bouchama A. Summer Hajj pilgrimage in the era of global warming: a call for vigilance and better understanding of the risks. J Travel Med. 2019;26(7):taz069. doi: 10.1093/jtm/taz069. [PubMed: 31518432].

12. Shinchi K, Ashida H. Proposal of a model for medical records for international disaster relief operations. Mil Med. 2003; 168(2):120-3. [PubMed: 12636139].

13. Luber G, McGeehin M. Climate change and extreme heat events. Am J Prev Med. 2008;35(5):429-35. doi: 10.1016/j.amepre. 2008.08.021. [PubMed: 18929969].

14. Glazer JL. Management of heatstroke and heat exhaustion. Am Fam Physician. 2005;71(11):2133-40. [PubMed: 15952443].

15. Doan Q, Wong H, Meckler G, Johnson D, Stang A, Dixon A, et al. The impact of pediatric emergency department crowding on patient and health care system outcomes: a multicentre cohort study. CMAJ. 2019;191(23):E627-35. doi: 10.1503/cmaj.181426. [PubMed: 31182457]. 